\title{
Two phase II trials of temozolomide with interferon- $\alpha 2 b$ (pegylated and non-pegylated) in patients with recurrent glioblastoma multiforme
}

\section{Groves*,', VK Puduvalli', MR Gilbert', VA Levin', CA Conrad', VH Liu', K Hunter', C Meyers', KR Hess², WK Alfred Yung'}

'Department of Neuro-Oncology, The University of Texas MD Anderson Cancer Center, Houston, TX, USA; ${ }^{2}$ Department of Biostatistics, The University of Texas MD Anderson Cancer Center, Houston, TX, USA

BACKGROUND: Because of the poor outcomes for patients with recurrent glioblastoma multiforme (GBM), and some laboratory and clinical evidence of efficacy using interferon in GBM, we assessed the toxicity and efficacy of temozolomide (TMZ) combined with either short-acting (IFN) or long-acting (pegylated) interferon $\alpha 2 b$ (PEG) in two single-arm phase II studies, and compared the results to 6-month progression-free survival (PFS-6) data from historical controls.

METHODS: Two single-arm phase II studies were carried out in adults with GBM. Patients were treated with the standard regimen of TMZ ( $150-200 \mathrm{mg} \mathrm{m}^{-2}$ per day $\times 5$ days every month) combined with either 4 million units per $\mathrm{m}^{2}$ subcutaneously (SQ) three times weekly of IFN or $0.5 \mu \mathrm{g} \mathrm{kg}^{-1} \mathrm{SQ}$ weekly of PEG. Physical exams and imaging evaluations were carried out every 8 weeks. RESULTS: On the IFN study, 34 adults (74\% men) were enrolled, and 29 adults (55\% men) on the PEG study; median Karnofsky performance status was 80 and 90 for the IFN and PEG studies, respectively. Grade 3 or 4 toxicities were common, leucopoenia and thrombocytopoenia occurring in 35-38\% and $18-21 \%$ of patients, respectively. Grade 3 or 4 fatigue occurred in 18\% of patients on both studies. Lymphopoenia was infrequent. PFS-6 was $31 \%$ for 29 evaluable patients in the IFN study and $38 \%$ for 26 evaluable patients in the PEG study.

CONCLUSION: In recurrent GBM patients, both studies of standard dose TMZ with either IFN or PEG showed improved efficacy when compared to historical controls, or reports using TMZ alone. Even though the TMZ + PEG study met criteria for further study, the results of both of these studies must be considered in light of the standard of care (TMZ plus radiotherapy) for newly diagnosed GBM, which has evolved since the inception of these studies. Despite the results of the current studies being eclipsed by the new GBM standard of care, these results can still inform the development of newer approaches for GBM, either in an earlier, upfront setting, or by extrapolation of the results and consideration of the use of PEG or IFN in conjunction with other antiglioma strategies. British Journal of Cancer (2009) I 0 I, 6I5-620. doi:I0.I038/sj.bjc.6605 I89 www.bjcancer.com

(c) 2009 Cancer Research UK

Keywords: glioblastoma; multiforme; temozolomide; interferon; phase II

Nearly 9000 persons every year are diagnosed with glioblastoma (GBM) (CBTRUS, 2002) in the United States, and most of them die within 15 months of diagnosis due to tumour progression. Deaths occur despite aggressive surgical resection, followed by the current standard of involved-field radiation plus temozolomide (TMZ) followed by adjuvant postradiation TMZ chemotherapy (Stupp et al, 2005). Glioblastomas contain numerous genetic and epigenetic alterations that allow for their initiation and resistance to treatment, but a complete understanding of GBM biology is lacking (Ohgaki and Kleihues, 2007). Due to the incomplete understanding of GBM biology, difficulties with central nervous system (CNS) drug delivery, lack of availability of specific and targeted drugs and other logistic factors, no targeted or cytotoxic agent has surfaced as a clearly effective treatment for recurrent GBM patients.

\footnotetext{
*Correspondence: Dr MD Groves; E-mail: mgroves@mdanderson.org Received 2 June 2009; revised I 8 June 2009; accepted 22 June 2009
}

Interferons (IFNs) are naturally occurring glycoproteins with immunomodulatory, antiproliferative, and antiangiogenic effects (Kirkwood and Ernstoff, 1984; Blanco et al, 2001; Jonasch and Haluska, 2001; Maher et al, 2007). The type I IFNs, $\alpha$ and $\beta$, are used in various malignant diseases (Maher et al, 2007). Numerous studies in glioma patients using both $\alpha$ and $\beta$ IFNs have demonstrated possible efficacy. In recurrent GBM patients treated with intravenous IFN $\beta$, combined response rates (objective response and stable disease) of $51 \%$ (Yung et al, 1991) were reported. Other trials using IFN $\beta$ have studied both recurrent GBM and postradiotherapy GBM patients who were stable (Fine et al, 1997; Colman et al, 2006) with suggestions of benefit. Varying degrees of efficacy have been demonstrated using IFN $\alpha$ in recurrent primary malignant gliomas, either alone or in combination with other modalities (Boethius et al, 1983; Hirakawa et al, 1983; Nakagawa et al, 1983; Nagai and Arai, 1984; Mahaley et al, 1985; Obbens et al, 1985; Hamada et al, 1986; Jereb et al, 1989; Yoshida et al, 1994; Buckner et al, 1995; Dillman et al, 1995; Brandes et al, 1997; Rajkumar et al, 1998). However, a large phase III trial compared postradiation BCNU either with or without IFN $\alpha$, and did not show 
a benefit with the addition of IFN $\alpha$ (Buckner et al, 2001). A recent report of a small number of GBM patients suggested a possible inverse correlation between IFN $\alpha$ gene content and response to high-dose IFN $\alpha$ (Olson et al, 2004).

IFN $\alpha 2 b$ is a highly purified human protein produced by recombinant DNA techniques with indications for several malignant diseases. A formulation of IFN $\alpha 2 b$ using polyethylene glycol (PEG-Intron, peg IFN $\alpha 2 b$ ) was developed to allow for longer patient exposure to drug. Conjugation of IFN $\alpha 2 \mathrm{~b}$ with polyethylene glycol significantly increases its half life $(t 1 / 2)$ from approximately 4.0 to 40.3 hours, allowing for once a week (QW) dosing.

Temozolomide is a well-tolerated, orally bioavailable alkylating agent, approved by the Food and Drug Administration (FDA) for the adjuvant treatment of patients with GBM during and after radiotherapy. This regimen resulted in a median survival of 14.6 months and a 2-year survival of $26 \%$ (Stupp et al, 2005). Before its approval in the newly diagnosed GBM, TMZ was commonly used as a cytotoxic agent in combination with a variety of other antiglioma agents.

Because of the previous data suggesting activity of IFNs in recurrent GBM, we undertook the studies reported here to determine whether IFN $\alpha 2 \mathrm{~b}$ (standard and pegylated formulation) along with TMZ at the standard 5-day dosing scheme could improve 6-month progression-free survival (PFS-6) in patients with recurrent GBM. As all type I IFNs bind to the same twosubunit receptor, no significant difference in activity was expected between IFN $\alpha$ or $-\beta$ (Maher et al, 2007). Interferon- $\alpha 2 \mathrm{~b}$ was chosen based on its broader history of use in malignant diseases, and on its ease of acquisition from the pharmaceutical sponsor of the studies. Results of two studies, TMZ plus IFN $\alpha 2 \mathrm{~b}$ (IFN) and TMZ plus pegylated IFN $\alpha 2 \mathrm{~b}$ (PEG) are presented, and both demonstrate benefit in prolonging PFS-6.

\section{PATIENTS AND METHODS}

\section{Inclusion criteria}

To be included in these studies, adult patients $(\geqslant 18$ years old) with a Karnofsky performance status (KPS) of $\geqslant 60 \%$ must have had intracranial GBM with evidence of tumour recurrence on CT or MRI scan of the brain. They must have failed previous radiation therapy (completed greater than 4 weeks before enrolment), and be on a stable dose of steroids. Patients may have had up to two previous chemotherapy regimens for not more than two previous relapses, and no exposure to either study drug. Patients were required to sign an institutional review board-approved informed consent, and to have recovered from effects of previous chemotherapy and have adequate organ function.

\section{Study design}

Treatment, doses, and evaluations Both studies were single-arm, open label phase II studies. Both studies used TMZ on a days $1-5$ of the 28-day schedule at a starting dose of $200 \mathrm{mg} \mathrm{m}^{-2}$ for patients not previously treated with any chemotherapy or at $150 \mathrm{mg} \mathrm{m}^{-2}$ for patients who had received previous chemotherapy. The earlier study used standard interferon $\alpha 2 \mathrm{~b}$ (IFN) and the later study used PEG. Interferon was dosed at 4 million units (MU) $\mathrm{m}^{-2}$ per day subcutaneously (SQ) 3 days per week (Monday, Wednesday, Friday), days 8 through 28 of each 28 -day course. PEG $0.5 \mu \mathrm{g} \mathrm{kg}^{-1}$ was administered by SQ injection every week. Treatment courses were repeated every 28 days if all toxicities from the previous course have resolved to grade 2 or less. For continuation on study drugs, patients were required to have recovered haematologically to an absolute neutrophil count of $\geqslant 1500$ per $\mu$, platelet count $\geqslant 100000$ per $\mu \mathrm{l}$, and all drug associated non-haematological toxicities had to have recovered to either grade 0 or 1 . Drugs were held until recovery from toxicity had occurred. If recovery had not occurred by day 28 , the subsequent course of TMZ was delayed until the toxicity criteria noted above were met. The dose of TMZ in subsequent courses was individually titrated and continued without interruption unless tumour recurrence or progression, and if toxicity was acceptable. Continuation of therapy after the first year was decided on an individual basis. For grade 3 or greater toxicities, treatment was withheld and the patients were monitored until toxicities resolved to grade 2 or better, then subsequent courses were started. A minimum of a 2 -week rest period was required for grade 3 or greater toxicity. Dosages for subsequent courses were one dose level below the dose that produced toxicity of grade 3 or greater. Dose modifications were allowed based on patient tolerance. PEG was maintained at the starting dose if well tolerated, but dose deescalation by $50 \%$ ( 0.25 and then to $0.125 \mu \mathrm{g} \mathrm{kg}^{-1}$ per week) was allowed. Interferon was started at $4 \mathrm{MU} \mathrm{m}^{-2}$ and could be increased to 5 or $6 \mathrm{MU} \mathrm{m}^{-2}$, or decreased to 3 or $2 \mathrm{MU} \mathrm{m}^{-2}$ as needed. Temozolomide could be deescalated to 175 or $150 \mathrm{mg} \mathrm{m}^{-2}$ (no previous TMZ exposure) or 125 or $100 \mathrm{mg} \mathrm{m}^{-2}$ (previous TMZ exposure) based on drug tolerance. Only two dose deescalations of IFN, PEG, or TMZ were permitted. Patients experiencing grade 3 or greater toxicities after two dose reductions were taken off the study. All patients were maintained on the lowest steroid dose necessary.

A minimum of 8 weeks of treatment was required for a patient to be considered as having received an adequate trial to evaluate efficacy. All patients were evaluable for toxicity and were followed for at least 30 days after completion of treatment. Any patient who progressed clinically during the first 8 weeks of therapy was evaluated by brain MRI scan and considered a protocol failure if disease progression was documented by neuroimaging. Patients who, for reasons other than clinical progression, discontinued treatment before the 8-week time point were not considered evaluable for efficacy of the treatment and not included in the PFS-6 determination.

Before enrolment, patients underwent a complete history and neurological examination, KPS determination, and documentation of evaluable disease. Pretreatment tests included CBC and standard blood tests verifying normal organ function, neuropsychological testing battery and brief fatigue inventory (only some patients), and contrast enhanced (Gd-DPTA) brain MRI scan. Baseline blood tests and MRI scans were performed within 14 days of registration.

During participation in the study, patients had routine blood testing (CBC and organ function tests) on day 22 of every 28-day course. Every 8 weeks, before odd-numbered courses, patients underwent contrast-enhanced brain MRI scans, physical and neurologic examinations with KPS and neuropsychological battery testing. A subset of patients also completed the brief fatigue inventory.

Outcome measures The primary endpoint of the study was PFS-6, but patients were also observed for response to treatment, using a combination of the neurological examination and MRI brain scan to define overall response or progression. Bidimensional measurable lesions were necessary for response evaluation according to the guidelines promulgated by Macdonald et al (1990), and were required to be maintained for at least 4 weeks. Responses were only declared if patients were on no or stable maintenance doses of corticosteroids, had stable or improved neurological exams, and were determined, based on MRI changes, to have a reduction in tumour size. A complete response (CR) was complete disappearance of all measurable and evaluable disease and no new lesions, and no evidence of non-evaluable disease. A partial response (PR) was defined as $\geqslant 50 \%$ decrease in the sum of products of perpendicular diameters of all measurable lesions, and no new lesions. Stable disease patients were those who did not qualify for $\mathrm{CR}, \mathrm{PR}$, or progression, and required a minimum of 12 weeks duration. Progression was declared when the MRI scan 
revealed a $\geqslant 25 \%$ increase in the sum of products of all measurable lesions over the smallest sum observed (over baseline if no decrease), or appearance of any new lesion/site, or failure to return for evaluation due to death or deteriorating condition. Although the neurological examination was not used for determining response, it was noted and recorded and used in conjunction with the imaging data to determine a patient's overall clinical status. Patients were described as normal, improved from on-study state, the same as on-study state, or worse than on-study state (patient worse). Time to treatment failure was determined from the date of registration to the date of first observation of progressive disease, non-reversible neurologic progression, death due to any cause, or early discontinuation of treatment. Survival was determined from the date of registration to the date of death (due to any cause). Steroid dosage was carefully monitored and recorded during each course of therapy, and steroid dosage changes were considered before response determinations are made. NCI Clinical Toxicity Criteria, applicable to the time frame for each study, was used for toxicity determinations.

\section{Statistical analysis}

The primary endpoint for both studies was improvement in PFS-6 (26 weeks). The historical values for comparison were from a database of 225 recurrent GBM patients enrolled in 8 previous phase II studies (in which none of the treatments were particularly effective; Wong et al, 1999). The proportion of patients remaining alive and free from progression at 6 months was $15 \%$ in that report.

Both studies were single arm by design. For the IFN study, hypotheses were $\mathrm{H}_{0}: P \leqslant P 0$ vs $\mathrm{H} 1: P \geqslant P 1$, where $P$ was the probability of remaining alive and free from progression at 6 months; $\alpha$ (false positive rate) was $=10 \%$ and $\beta$ (false negative rate) $=5 \%$. $P 0$ was set to $15 \%$ (95\% CI for the proportion alive and FFP at 6 months was from 17 to $26 \%$ ). p 1 was set to $35 \%$, looking for an improvement of 0.2 . The plan was to declare success if $>22.5 \%$ of patients were alive and free from progression at 6 months, with an $\alpha$ of $7 \%$ and a $\beta$ of $6 \%$. A 35\% reponse (PFS-6) would yield a $95 \%$ CI on the true response proportion from 21 to $52 \%$.

For the PEG study, a Simon's optimal two-stage design was employed. The hypotheses to be tested were $\mathrm{H}_{0}: p \leqslant p 0$ vs $\mathrm{H}_{1}$ : $p \geqslant p 1$, where $p$ is the probability of remaining alive and free from progression at 6 months; $\alpha$ was $10 \%$, and $\beta$ was $5 \%$. $p 0$ was set to $10 \%$ (The $95 \%$ confidence interval for our estimate of the proportion alive and progression free at 6 months was from 10 to $19 \%$.) $p 1$ was set to $30 \%$ (looking for an improvement of 0.2 ). These parameters led to a trial with a first stage of 20 patients (stopping if 2 or fewer patients respond) and a total of 40 patients (declaring success if more than 6 patients respond). As the outcome was observed over a 6-month period, it was necessary to suspend enrolment after the first 20 patients were enrolled to determine if the trial should be stopped or continued.

\section{RESULTS}

\section{Patients}

For the earlier IFN study, 34 GBM patients were enrolled in 1998 in less than 5.5 months. For the later PEG study, 29 GBM patients were enrolled over 39 months from September 2002 through December 2005. In the PEG study, accrual was halted because of the establishment of the use of temozolomide as first line treatment, markedly reducing the number of potentially eligible patients. Median time from completion of radiation to enrolment was 24 weeks (range 4-456 weeks) for the IFN study and 22 weeks (range 4-324 weeks) for the PEG study. In the IFN study, 9 of 34
Table I Patient demographics

\begin{tabular}{lcc}
\hline Parameter & TMZ+IFN & TMZ+PEG \\
\hline Number of patients & 34 & 29 \\
Age: median (range) & $55(17-69)$ & $56(20-67)$ \\
Gender: M/F (\%) & $25: 9(74: 26)$ & $16: 13(55: 45)$ \\
KPS & & \\
Median & 80 & 90 \\
100\% & $3(9 \%)$ & $5(17 \%)$ \\
$90 \%$ & $7(21 \%)$ & $16(55 \%)$ \\
$80 \%$ & $17(50 \%)$ & $5(17 \%)$ \\
$70 \%$ & $7(21 \%)$ & $3(10 \%)$ \\
& & \\
Maximum prior resection & $2(6 \%)$ & $5(17 \%)$ \\
Biopsy & $20(59 \%)$ & $9(31 \%)$ \\
Subtotal resection & $12(35 \%)$ & $15(52 \%)$ \\
Gross total resection & & $14(48 \%)$ \\
Number of prior chemotherapies & $9(26 \%)$ & $14(48 \%)^{\mathrm{b}}$ \\
0 & $24(71 \%)^{\mathrm{a}}$ & $1(3 \%)$ \\
1 & $1(3 \%)$ & \\
2 &
\end{tabular}

aPrior chemotherapies included: topotecan, BCNU, cisplatinum, procarbazine+ CCNU+vincristine (PCV), hydroxyurea, marimastat, 6-thioguanine, 13-cis-retinoic acid, carboplatin, and etoposide. ${ }^{b}$ Prior chemotherapies included: celecoxib, gefitinib, $\mathrm{BCNU}, \mathrm{CCNU}$, vincristine, 13 -cis-retinoic acid, PCV, tipifarnib, and erlotinib.

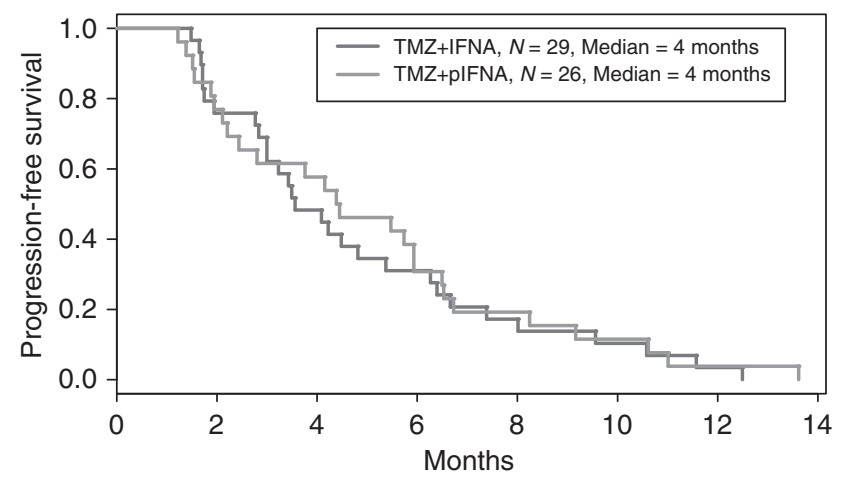

Figure I PFS-6 results for TMZ + IFN and TMZ + PEG.

(26\%) patients were enrolled within 3 months of completing radiation and for the PEG study, this figure was 7 of $29(24 \%)$. Details of patient demographics are set forth in Table 1. There were differences between the two groups in the IFN and PEG studies, but using multivariable logistic regression analysis, no statistically significant differences were identified between the IFN and PEG groups with respect to age at diagnosis, KPS at enrolment, gender, previous extent of resection, or number of previous chemotherapies (all results: $P>0.05$ ).

\section{Response and survival}

Eight patients were not evaluable for response and these patients were not included in the survival analysis. One patient never received treatment (IFN study), two developed intercurrent illnesses that caused treatment discontinuance before treatment 1 course was administered (both in the PEG study) and five were lost to follow-up (four from the IFN study and one from the PEG study). Six-month progression-free survival was $31 \%$ for 29 evaluable patients in the IFN group and $38 \%$ for 26 evaluable patients in the PEG group. Kaplan-Meier survival curves for PFS-6 are shown in Figure 1. Results for patients enrolled within the first 3 months after completing radiation (patients with 
Table 2 Response data

\begin{tabular}{|c|c|c|c|c|c|c|}
\hline Study & $\begin{array}{c}\text { 6-Month PFS \% } \\
(95 \% \mathrm{CI})\end{array}$ & $\begin{array}{l}\text { Median PFS months } \\
\text { (95\% CI) }\end{array}$ & $\begin{array}{l}\text { Median OS months } \\
(95 \% \mathrm{Cl})\end{array}$ & $\begin{array}{l}\text { \% Responders } \\
\text { (all partial) }\end{array}$ & $\begin{array}{l}\% \text { Stable } \\
\text { disease }\end{array}$ & $\begin{array}{c}\% \text { Responders+stable } \\
\text { disease }\end{array}$ \\
\hline $\mathrm{TMZ}+\mathrm{IFN} ; n=34$ & $31(16-54)^{a}$ & $3.6(3.0-6.3)$ & $7.2(5.3-10.6)$ & $4 / 34(12 \%)$ & | 8/34 (53\%) & $22 / 34(65 \%)$ \\
\hline TMZ+PEG; $n=29$ & $38(24-64)^{a}$ & $4.4(2.4-6.5)$ & $10.0(7.8-14.3)$ & $1 / 29(3 \%)$ & $17 / 29$ (57\%) & $18 / 29(62 \%)$ \\
\hline
\end{tabular}

Numbers are $n=29$ for TMZ+IFN and $n=26$ for TMZ+PEG due to patients being not evaluable for response.

Table 3 Grade 3 and 4 adverse events

\begin{tabular}{lcc}
\hline Grade 3 or $\mathbf{4}$ toxicity & $\begin{array}{c}\text { IFN study (N=33): } \\
\boldsymbol{n}(\%)\end{array}$ & $\begin{array}{c}\text { PEG study (N=29): } \\
\mathbf{n}(\%)\end{array}$ \\
\hline Leucopoenia & $12(35)$ & $11(38)$ \\
Thrombocytopoenia & $6(18)$ & $6(21)$ \\
Fatigue & $6(18)$ & $6(18)$ \\
Pulmonary embolus/thrombosis & $0(0)$ & $5(17)$ \\
Nausea/vomiting & $2(6)$ & $0(0)$ \\
Headache & $0(0)$ & $2(7)$ \\
Incontinence & $0(0)$ & $2(7)$ \\
Other & $2(3)^{\mathrm{a}}$ & $8(3)^{\mathrm{b}}$ \\
\hline
\end{tabular}

ane instance each of diarrhoea and elevated transaminases. ${ }^{b}$ One instance each of anaemia, confusion, depression, hypotension, hypoxemia, infection, lymphopoenia, and pruritis.

possible pseudoprogression) were a PFS- 6 of $12.5 \%$ for the IFN study $(n=9)$ and $57 \%$ for the PEG study $(n=7)$. Excluding those patients enrolled within 3 months after completing radiation, leaves the IFN study with a PFS- 6 of $40 \%$ and the PEG study with a PFS- 6 of $35 \%$. Additional response and PFS data are presented in Table 2. Using multivariate Cox proportional hazard regression analysis, the hazard ratio for progression for the PEG group was 0.63 (95\% confidence interval: $0.3,1.2, P=0.15)$. Due to wide a confidence interval, these results are inconclusive.

\section{Adverse events}

All patients except the one from the IFN study who received no treatment are included in the report of toxicities. In the IFN and PEG groups, 20 of $33(60 \%)$ and 21 of $29(72 \%)$, respectively, of patients suffered at least one grade 3 or 4 toxicity event. Details of the grade 3 and 4 toxicities are presented in Table 3. Of those patients who had not received previous chemotherapy, 7 of 23 (30\%) suffered grade 3 or greater thrombocytopoenia compared to 4 of $40(10 \%)$ of those who had previously received chemotherapy. Grade 3 or greater leucopoenia occurred in 9 of $23(39 \%)$ and 13 of $40(33 \%)$ of patients who had no previous chemotherapy $v s$ those who had received previous chemotherapy, respectively. Thus, previous exposure to chemotherapy did not appear to increase the risk of grade 3 or greater haematotoxicity in either study. However, this may have been due to lower TMZ dosing in those patients with previous chemotherapy exposure.

\section{Neurocognitive testing}

Only 11 patients from the PEG study had baseline and at least 2 follow-up neurocognitive assessments. Very little cognitive decline was seen in these patients, but the quantity of data was felt insufficient from which to model statistically significant changes or make conclusions.

\section{DISCUSSION}

The current studies were conceived and their enrolment initiated before FDA approval of TMZ for GBM patients. The 2005 FDA approval of TMZ for GBM in the upfront setting, and the subsequent widespread adoption of this regimen (Stupp et al, 2005) resulted in the closure of the PEG study because of low availability of non-TMZ-treated patients and slow accrual. In the future, studies using modified dosing schedules of TMZ and/or combinations of TMZ with newer agents designed to overcome TMZ resistance might be necessary to ensure adequate accrual. Additionally, because of the use of TMZ with radiotherapy in the upfront setting in GBM, these results are somewhat anachronistic. But this fact does not diminish the study results when compared to the older data. The new data can serve as building blocks for future studies of interferons in different settings in glioma.

Despite the incomplete accrual of the second study, we believe these results provide interesting information, especially when compared to single-agent TMZ use and to the historical data. Based on the original statistical designs, the PEG study, but not the IFN study, met criteria as potentially worthy of further study. The PFS- 6 results of 31 and $38 \%$ for IFN and PEG, respectively, compare favourably to the PFS- 6 of $21 \%$ reported with standard (5-day every 28 days) dose TMZ in recurrent GBM (Yung et al, 2000). Even though the demographic differences (higher enrolment KPS, increased percentage of gross total resection, and lower number of previous chemotherapies) between the patients in the PEG $v s$ IFN group did not meet statistical significance, these differences, favouring the PEG group, could have impacted outcomes.

The reasons for the improved outcomes reported here are not known, although some hypotheses can be offered. The short arm of chromosome 9, which contains the IFN gene cluster, is commonly lost in both primary and secondary GBMs (Maher et al, 2006), as well as in recurrent high-grade tumours when compared to low-grade tumours (Kim et al, 1995; Weber et al, 1996). There is evidence that the short arm of chromosome 9 may contains genes (IFN or others) with tumour suppressor activity (James et al, 1991, 1993). Malignant glioma specimens are reported to have a $50 \%$ rate of deletion of the IFN $\alpha$ gene cluster (James et al, 1991). Further, IFN $\alpha$ and $-\beta$ gene deletions are reported in $26-46 \%$ of glioma cell lines (Miyakoshi et al, 1990; James et al, 1993). These data notwithstanding, in vitro experiments suggest an antineoplastic action of IFN treatment, regardless of the IFN gene status of tumour cells (Miyakoshi et al, 1990). Replacement of IFN protein may, through a variety of mechanisms, result in an antitumour effect. Interferon- $\alpha$ results in both direct and indirect immunemediated effects (Barth and Morton, 1995), which may be relevant to the effects seen here. Antiproliferative, antiangiogenic, and prodifferentiation effects may also be contributing to the effects seen. Some evidence also suggests IFNs may downregulate MGMT, increasing tumour cell sensitivity to TMZ (Natsume et al, 2005).

A few features of the adverse event profile of these drug combinations warrant discussion. First, the risk of grade 3 or 4 anaemia and leucopoenia are almost equivalent to single-agent TMZ (temozolomide package insert). However, the risk of lymphopoenia $(3 \%)$ was remarkably lower than that reported for single-agent TMZ (55\%). Further, no pneumocystis pneumonia was observed in these patients. Additionally, the risk of thrombotic events was higher in the PEG-treated patients (17\%), as compared to the IFN-treated ones $(0 \%)$. The reasons for these observations 
are not clear from the literature as well as after review of the specific patient histories.

Even though the studies reported here appear to show some benefit over standard dose TMZ in recurrent GBM, several factors may militate against these results. As both studies are single institution and non-randomised, their results are less reliable and may reflect a referral bias. In addition, pseudoprogression at the time of enrolment in some patients cannot be completely excluded as a possible contributor to the improved outcomes, despite its apparent lack of impact when one excludes from analysis those patients enrolled within 3 months after the completion of radiotherapy.

\section{CONCLUSIONS}

The two phase II studies demonstrated some improvement in PFS-6 outcomes in recurrent GBM patients as compared with historical data and with the single-agent TMZ data. The PEG study met criteria to be deemed worthy of further investigation. The use of this combination and dosing scheme in the recurrent setting is probably not feasible at this time, due to the near universal exposure of GBM patients to TMZ as an up-front treatment. Possible future avenues of investigation of PEG in malignant glioma patients could include (1) PEG combined with TMZ and XRT in the up-front GBM setting (using MGMT methylation status or IFN $\alpha$ gene content as an enrolment criteria), (2) the addition of IFN or PEG to an intensified TMZ regimen at recurrence (Wick et al, 2007), (3) the addition of PEG to alternative cytotoxic chemotherapies, looking for improvement in outcomes, or (4) the addition of PEG to other non-cytotoxic therapies, as a potential synergistic agent.

\section{ACKNOWLEDGEMENTS}

We gratefully acknowledge the efforts of Sandra Ictech with data management for these studies. This study was supported by Schering Plough Research Institute

\section{REFERENCES}

Barth A, Morton DL (1995) The role of adjuvant therapy in melanoma management. Cancer 75: $726-734$

Blanco P, Palucka AK, Gill M, Pascual V, Banchereau J (2001) Induction of dendritic cell differentiation by IFN-alpha in systemic lupus erythematosus. Science 294: 1540 - 1543

Boethius J, Blomgren H, Collins VP, Greitz T, Strander H (1983) The effect of systemic human interferon-alpha administration to patients with glioblastoma multiforme. Acta Neurochir (Wien) 68: 239-251

Brandes AA, Scelzi E, Zampieri P, Rigon A, Rotilio A, Amista P, Berti F, Fiorentino MV (1997) Phase II trial with BCNU plus alpha-interferon in patients with recurrent high-grade gliomas. Am J Clin Oncol 20: 364-367

Buckner JC, Brown LD, Kugler JW, Cascino TL, Krook JE, Mailliard JA, Kardinal CG, Tschetter LK, O’Fallon JR, Scheithauer BW (1995) Phase II evaluation of recombinant interferon alpha and BCNU in recurrent glioma. J Neurosurg 82: $430-435$

Buckner JC, Schomberg PJ, McGinnis WL, Cascino TL, Scheithauer BW, O'Fallon JR, Morton RF, Kuross SA, Mailliard JA, Hatfield AK, Cole JT, Steen PD, Bernath AM (2001) A phase III study of radiation therapy plus carmustine with or without recombinant interferon-alpha in the treatment of patients with newly diagnosed high-grade glioma. Cancer 92: $420-433$

CBTRUS (2002) Statistical Report: Primary Brain Tumors in the United States, 1995-1999 Published by the Central Brain Tumor Registry of the United States

Colman H, Berkey BA, Maor MH, Groves MD, Schultz CJ, Vermeulen S, Nelson DF, Mehta MP, Yung WK, Radiation Therapy Oncology Group (2006) Phase II Radiation Therapy Oncology Group trial of conventional radiation therapy followed by treatment with recombinant interferonbeta for supratentorial glioblastoma: results of RTOG 9710. Int J Radiat Oncol Biol Phys 66: $818-824$

Dillman RO, Wiemann M, Oldham RK, Soori G, Bury M, Hafer R, Church C, DePriest C (1995) Interferon alpha-2a and external beam radiotherapy in the initial management of patients with glioma: a pilot study of the National Biotherapy Study Group. Cancer Biother 10: 265-271

Fine HA, Wen PY, Robertson M, O’Neill A, Kowal J, Loeffler JS, Black PM (1997) A phase I trial of a new recombinant human beta-interferon (BG9015) for the treatment of patients with recurrent gliomas. Clin Cancer Res 3: $381-387$

Hamada H, Asakura T, Maeda Y, Yokoyama S, Niiro M (1986) [A study on the direct antitumoral effect of interferon-alpha on human glioma]. Gan To Kagaku Ryoho 13: 464-471

Hirakawa K, Ueda S, Nakagawa Y, Suzuki K, Fukuma S, Kita M, Imanishi J, Kishida T (1983) Effect of human leukocyte interferon on malignant brain tumors. Cancer 51: 1976-1981

James CD, He J, Carlbom E, Nordenskjold M, Cavenee WK, Collins VP (1991) Chromosome 9 deletion mapping reveals interferon alpha and interferon beta-1 gene deletions in human glial tumors. Cancer Res 51: $1684-1688$
James CD, He J, Collins VP, Allalunis-Turner MJ, Day III RS (1993) Localization of chromosome 9p homozygous deletions in glioma cell lines with markers constituting a continuous linkage group. Cancer Res 53: $3674-3676$

Jereb B, Petric J, Lamovec J, Skrbec M, Soss E (1989) Intratumor application of human leukocyte interferon-alpha in patients with malignant brain tumors. Am J Clin Oncol 12: 1-7

Jonasch E, Haluska FG (2001) Interferon in oncological practice: review of interferon biology, clinical applications, and toxicities. Oncologist 6: $34-55$

Kim DH, Mohapatra G, Bollen A, Waldman FM, Feuerstein BG (1995) Chromosomal abnormalities in glioblastoma multiforme tumors and glioma cell lines detected by comparative genomic hybridization. Int $J$ Cancer 60: $812-819$

Kirkwood JM, Ernstoff MS (1984) Interferons in the treatment of human cancer. J Clin Oncol 2: 336-352

Macdonald DR, Cascino TL, Schold Jr SC, Cairncross JG (1990) Response criteria for phase II studies of supratentorial malignant glioma. J Clin Oncol 8: $1277-1280$

Mahaley Jr MS, Urso MB, Whaley RA, Blue M, Williams TE, Guaspari A, Selker RG (1985) Immunobiology of primary intracranial tumors. Part 10: Therapeutic efficacy of interferon in the treatment of recurrent gliomas. J Neurosurg 63: 719-725

Maher EA, Brennan C, Wen PY, Durso L, Ligon KL, Richardson A, Khatry D, Feng B, Sinha R, Louis DN, Quackenbush J, Black PM, Chin L, DePinho RA (2006) Marked genomic differences characterize primary and secondary glioblastoma subtypes and identify two distinct molecular and clinical secondary glioblastoma entities. Cancer Res 66: $11502-11513$

Maher SG, Romero-Weaver AL, Scarzello AJ, Gamero AM (2007) Interferon: cellular executioner or white knight? Curr Med Chem 14: 1279-1289

Miyakoshi J, Dobler KD, Allalunis-Turner J, McKean JD, Petruk K, Allen PB, Aronyk KN, Weir B, Huyser-Wierenga D, Fulton D, Urtasun RC, Day III, RS (1990) Absence of IFNA and IFNB genes from human malignant glioma cell lines and lack of correlation with cellular sensitivity to interferons. Cancer Res 50: $278-283$

Nagai M, Arai T (1984) Clinical effect of interferon in malignant brain tumours. Neurosurg Rev 7: 55-64

Nakagawa Y, Hirakawa K, Ueda S, Suzuki K, Fukuma S, Kishida T, Imanishi J, Amagai T (1983) Local administration of interferon for malignant brain tumors. Cancer Treat Rep 67: 833-835

Natsume A, Ishii D, Wakabayashi T, Tsuno T, Hatano H, Mizuno M, Yoshida J (2005) IFN-beta down-regulates the expression of DNA repair gene MGMT and sensitizes resistant glioma cells to temozolomide. Cancer Res 65: 7573-7579

Obbens EA, Feun LG, Leavens ME, Savaraj N, Stewart DJ, Gutterman JU (1985) Phase I clinical trial of intralesional or intraventricular leukocyte interferon for intracranial malignancies. J Neurooncol 3: 61-67 
Ohgaki H, Kleihues P (2007) Genetic pathways to primary and secondary glioblastoma. Am J Pathol 170: 1445-1453

Olson JJ, James CD, Lawson D, Hunter S, Tang G, Billingsley J (2004) Correlation of the response of recurrent malignant gliomas treated with interferon alpha with tumor interferon alpha gene content. Int J Oncol 25: $419-427$

Rajkumar SV, Buckner JC, Schomberg PJ, Cascino TL, Burch PA, Dinapoli RP (1998) Phase I evaluation of radiation combined with recombinant interferon alpha-2a and BCNU for patients with high-grade glioma. Int $J$ Radiat Oncol Biol Phys 40: 297-302

Stupp R, Mason WP, van den Bent MJ, Weller M, Fisher B, Taphoorn MJ, Belanger K, Brandes AA, Marosi C, Bogdahn U, Curschmann J, Janzer RC, Ludwin SK, Gorlia T, Allgeier A, Lacombe D, Cairncross JG, Eisenhauer E, Mirimanoff RO, European Organisation for Research and Treatment of Cancer Brain Tumor and Radiotherapy Groups; National Cancer Institute of Canada Clinical Trials Group (2005) Radiotherapy plus concomitant and adjuvant temozolomide for glioblastoma. $N$ Engl J Med 352: 987-996

Weber RG, Sabel M, Reifenberger J, Sommer C, Oberstrass J, Reifenberger G, Kiessling M, Cremer T (1996) Characterization of genomic alterations associated with glioma progression by comparative genomic hybridization. Oncogene 13: 983-994
Wick A, Felsberg J, Steinbach JP, Herrlinger U, Platten M, Blaschke B, Meyermann R, Reifenberger G, Weller M, Wick W (2007) Efficacy and tolerability of temozolomide in an alternating weekly regimen in patients with recurrent glioma. J Clin Oncol 25: 3357-3361

Wong ET, Hess KR, Gleason MJ, Jaeckle KA, Kyritsis AP, Prados MD, Levin VA, Yung WK (1999) Outcomes and prognostic factors in recurrent glioma patients enrolled onto phase II clinical trials. J Clin Oncol 17: 2572-2578

Yoshida J, Kajita Y, Wakabayashi T, Sugita K (1994) Long-term follow-up results of 175 patients with malignant glioma: importance of radical tumour resection and postoperative adjuvant therapy with interferon, ACNU and radiation. Acta Neurochir (Wien) 127: 55-59

Yung WK, Albright RE, Olson J, Fredericks R, Fink K, Prados MD, Brada M, Spence A, Hohl RJ, Shapiro W, Glantz M, Greenberg H, Selker RG, Vick NA, Rampling R, Friedman H, Phillips P, Bruner J, Yue N, Osoba D, Zaknoen S, Levin VA (2000) A phase II study of temozolomide vs. procarbazine in patients with glioblastoma multiforme at first relapse. Br J Cancer 83: 588-593

Yung WK, Prados M, Levin VA, Fetell MR, Bennett J, Mahaley MS, Salcman M, Etcubanas E (1991) Intravenous recombinant interferon beta in patients with recurrent malignant gliomas: a phase I/II study. J Clin Oncol 9: 1945-1949 